05,12

\title{
Synthesis, Characterization, and Magnetic Behavior of Cobalt-Ferrite Nanoparticles under Variant Temperature Conditions
}

\author{
(C) M.A. Alotaibi ${ }^{1}$, I. Ud Din ${ }^{1,}$, A.I. Alharthi ${ }^{1}$, P. Ahmad ${ }^{2}$, A. Naeem ${ }^{3}$, I.A. ElSayed ${ }^{1}$, G. Centi ${ }^{4}$ \\ ${ }^{1}$ Department of Chemistry, College of Science and Humanities, Prince Sattam Bin Abdulaziz University, \\ P.O. Box 173, Al-Kharj 11942, Saudi Arabia \\ ${ }^{2}$ Department of Physics, University of Azad Jammu \& Kashmir, \\ 13100 Muzaffarabad, Pakistan \\ ${ }^{3}$ National Centre of Excellence in Physical Chemistry, University of Peshawar, \\ Pakistan \\ ${ }^{4}$ University of Messina, ERIC aisbl and CASPE/INSTM, Departments ChiBioFarAm and MIFT, \\ viale F. Stagno d'Alcontres 31, 98166 Messina, Italy \\ IE-mail: drisraf@yahoo.com
}

Received: November 11, 2020

Revised: November 11, 2020

Accepted: November 20, 2020

Wet chemical method was applied for the synthesis of cobalt-ferrite nanoparticles. The physicochemical properties were investigated by number of analytical techniques. TGA revealed the thermal stability of synthesized cobaltferrite nanoparticles. $X$-ray diffraction studies displayed the nanoparticles crystalline nature. Structure of cobaltferrite nanoparticles was confirmed via infrared spectroscopy by manifesting Co and $\mathrm{Fe}$ ions absorption peaks. Morphological studies showed synthesis of nanoparticles of cobalt-ferrite by employing field emissions scanning electron microscopy. The magnetic properties of cobalt-ferrite nanoparticles were investigated by vibrating sample magnetometer (VSM). The X-ray photoelectron spectroscopy studies confirmed the synthesis of cobalt-ferrite by displaying the oxidation of $\mathrm{Co}$ as $\mathrm{Co}^{2+}$ and $\mathrm{Fe}$ as $\mathrm{Fe}^{3+}$, respectively. The VSM results revealed that the magnetic characteristics of cobalt-ferrite nanoparticles were completely changed by the variation of temperature.

Keywords: ferrite nanoparticles, VSM, temperature effect, magneton number, anisotropy constant. 